\title{
A retrospective study on incidence of lameness in domestic animals
}

\author{
A. Mohsina ${ }^{1}$, M. M. S Zama ${ }^{1}$, P. Tamilmahan ${ }^{1}$, M. B. Gugjoo ${ }^{1}$, K. Singh ${ }^{1}$, A.Gopinathan ${ }^{1}$, M. Gopi ${ }^{2}$ and K. Karthik ${ }^{3}$ \\ 1. Division of Veterinary Surgery, Indian Veterinary Research Institute, Izatnagar, Bareilly, 243 122, Uttar Pradesh, India; \\ 2. Division of Animal Nutrition, Indian Veterinary Research Institute, Izatnagar, Bareilly, 243 122, Uttar Pradesh, India; \\ 3. Division of Bacteriology, Indian Veterinary Research Institute, Izatnagar, Bareilly, 243 122, Uttar Pradesh, India; \\ Corresponding author: A. Mohsina, email: sowparnika777@gmail.com, Cell: +91-9458587867 \\ MMSZ: mmszama@yahoo.com,PT: drtamilmahan.bison@gmail.com,MBG: mbgugjoo@gmail.com, \\ KS: ksuppli@yahoo.co.in, AG: aswathykiran77@gmail.com, MG: gopsgopi72@gmail.com, KK: karthik_2bvsc@yahoo.co.in \\ Received: 23-04-2014, Revised: 28-06-2014, Accepted: 05-07-2014, Published online: 15-08-2014
}

doi: 10.14202/vetworld.2014.601-604

How to cite this article: Mohsina A, Zama MMS, Tamilmahan P, Gugjoo MB, Singh K, Gopinathan A, Gopi M and Karthik K (2014) A retrospective study on incidence of lameness in domestic animals, Veterinary World 7(8): 601-604.

\begin{abstract}
Aim: To study the incidence of lameness among different species of animals presented to the Veterinary Polyclinic, Indian Veterinary Research Institute, Izatnagar.

Materials and Methods: Outpatient department (OPD) records for the period from January 2006 to December 2010 were referred and information was collected regarding number of lameness in different species, breeds, type of injury, limb affected, gender, age at onset, treatment offered, outcome and any reoccurrence. In this study, fractured cases were not included.

Results: The incidence of lameness among different species were recorded: canine (56\%), equine (21\%), caprine ( $7 \%$ ), feline $(3 \%)$, cattle $(7 \%)$, buffalo $(5.47 \%)$, sheep $(0.6 \%)$, monkey $(0.39 \%)$ and swine $(0.19 \%)$. In dog, the different conditions were reported with hind quarter weakness recording $55 \%$ of lameness followed by right hind limb lameness (14.7\%), left hind limb lameness $(12.6 \%)$, left forelimb lameness (12\%), hip dislocation $(6.3 \%)$ and hip dysplasia (4.2\%). In caprines, important causes of lameness were right forelimb lameness (23\%), right hind limb lameness (12\%), left forelimb lameness (12\%), posterior paresis (9\%), left shoulder dislocation (14\%) and right shoulder dislocation (6\%). In cattle, $34.28 \%$ of cases with right hind limb lameness, $28.5 \%$ cases were due to HQW, $14.28 \%$ had hip dysplasia, $8.57 \%$ suffered left hind limb lameness, $6 \%$ cases were recorded with obturator nerve paralysis and $8.57 \%$ cases suffered contracted tendon in calves. In buffaloes, cases reported were right carpal arthritis, foot rot and left hind limb lameness (14.28\%) each, due to bilateral upward luxation of patella and due bilateral purulent wound in stifle (18\%) each and hip dislocation (21.4\%). In equines, lameness were reported with right hind limb affection (13\%), left forelimb affection (11\%), right forelimb affection (17\%), 4\% each due to disease of right shoulder, HQW and both forelimb affection, lateral dislocation of patella (3\%), affection of both hind limbs $(9 \%), 5.5 \%$ each due to tenosynovitis, arthritis and bone spavin, $7 \%$ due to laminitis of right hind and forelimbs and $4.5 \%$ due to quittor.
\end{abstract}

Conclusion: The results show that lameness in animals is significantly affected by species, breed, age and sex of the animal. In dogs and cats, HQW is the most common cause of lameness. Among dogs, Mongrel and GSD breeds most affected. Male animals of less than 1 year or above 6 years are affected more. Hip dislocation is found to be more in male GSD and Labrador dogs and bilateral hip dislocation in spitz breeds. Hip dysplasia is also found to be more in GSD breed. Incidence of rickets is more in spitz dogs below 5 months of age. In equine and caprine species, right forelimb lameness, and in cattle right hind limb lameness is more. Hip dislocation, upward luxation of patella and wound are the major lameness causes in buffaloes.

Keywords: domestic animals, incidence, lameness.

Abbreviations: HQW- hind quarter weakness, GSD- German Shepherd, OPD- outpatient department, RHLL - right hindlimb lameness, LHLL - left hindlimb lameness, RFLL - right forelimb lameness, LFLL - left forelimb lameness, BFLL - bilateral forelimb lameness, BHLL - bilateral hindlimb lameness, HD - hip dislocation, Hip dysp - hip dysplasia, LDP - lateral dislocation of patella , RS - right shoulder, SD - shoulder dislocation, PP - posterior paralysis, Ob.N.P - obturator nerve paralysis, CT - contracted tendon.

\section{Introduction}

Lameness is defined as an abnormal stance or gait caused by either a structural or a functional disorder of the locomotor system [1]. A variety of reasons for lameness have been reported, including heritable defects, congenital defects, developmental defects, growth defects, trauma, and infection. Lameness is very common in dogs due to their active nature. Mus-

Copyright: The authors. This article is an open access article licensed under the terms of the Creative Commons Attribution License (http://creativecommons.org/licenses/by/2.0) which permits unrestricted use, distribution and reproduction in any medium, provided the work is properly cited. culoskeletal disorders are found to be more common cause than neurological disorder in dogs [2]. Shifting lameness was reported in dogs diagnosed for Lyme disease [3]. Age, sex and breed of the animals also influence the type and cause of lameness. In dairy cattle, main cause of lameness is pain in the limb, especially in the foot; hind feet are found to be more affected than front feet [4]. In piglets, rough and abrasive floors are found to be an important cause of lameness [5]. Lameness affects the viability, growth, reproduction and cause severe economic loss in pigs [6]. In cattle, economic loss can result from reduced 
Table-1: Incidence of lameness reported in different species during the study.

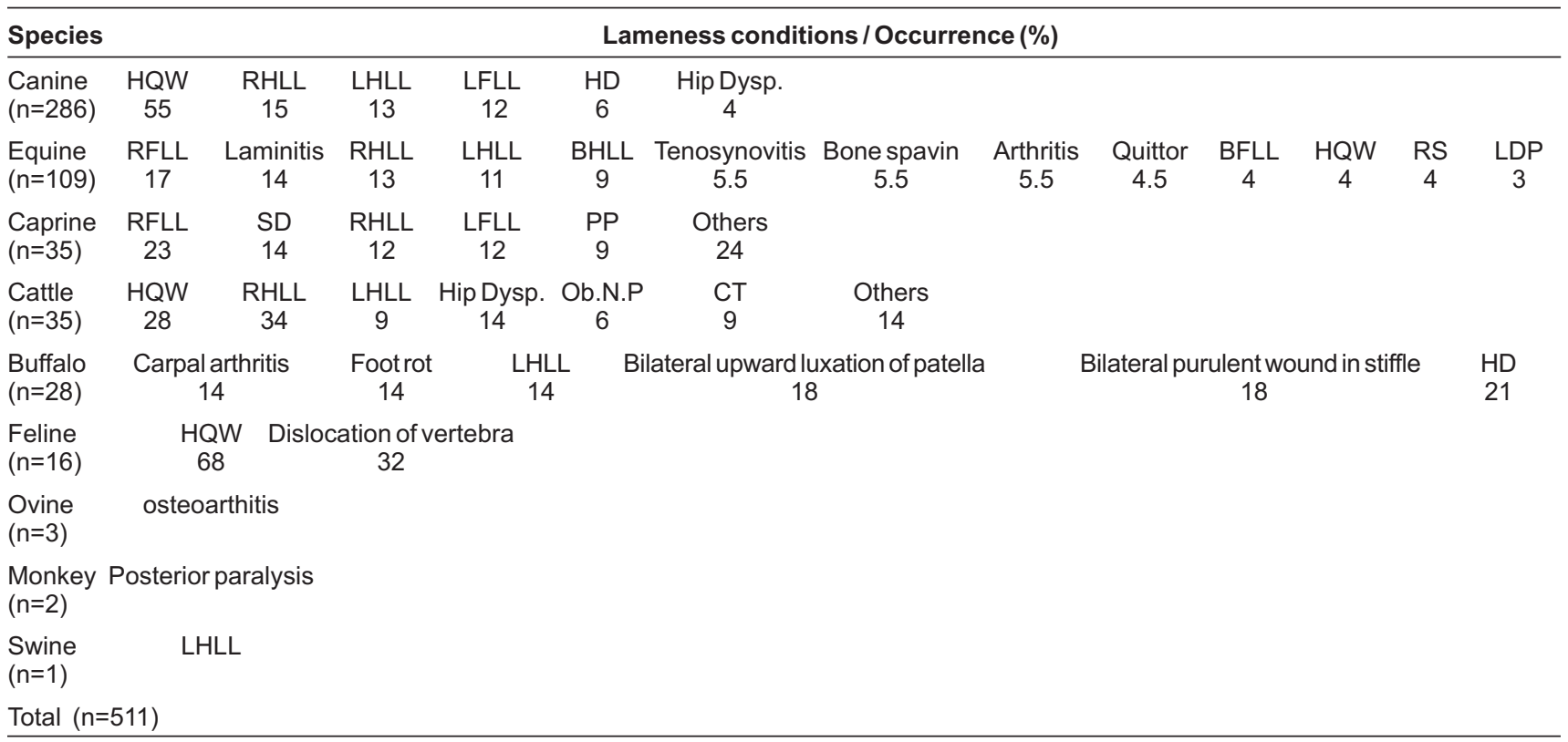

HQW- hind quarter weakness, RHLL - right hindlimb lameness, LHLL - left hindlimb lameness, LFLL - left forelimb lameness, HD - hip dislocation, Hip dysp - hip dysplasia, RFLL - right forelimb lameness, BHLL - bilateral hindlimb lameness, BFLL bilateral forelimb lameness, RS - right shoulder, LDP - lateral dislocation of patella, SD - shoulder dislocation, PP - posterior paralysis, Ob.N.P - obturator nerve paralysis, CT - contracted tendon.

milk yield, weight loss, disposal, deaths and replacement cost, infertility, prolonged calving interval, veterinary expenses and additional stockman's time [7].

Diagnosis of lameness is complex as it affects animal's ability to move and may be due to lesion of at least three body systems (musculoskeletal, nervous and integumentary) independently or in combination [6]. A structured approach to investigation of lameness is vital for treating the animal appropriately [2]. Thorough investigation of injured tissue will aid in the development of specific treatment plan [2].

This study reports the incidence of lameness except fracture cases in domestic animals for a period of five years.

\section{Materials and Methods}

Ethical approval: The study conducted complies with the guidelines laid down by the Institutional Ethical Committee.

A study was conducted to acquire detailed information regarding the cases of lameness reported among different species of animals which are referred to Division of Surgery, Indian Veterinary Research Institute (IVRI), OPD records for five years from January 2006 to December 2010 were referred and information were collected regarding number of lame animals, type of injury, limb affected, gender affected, age at onset, outcome, and if there was any reoccurrence. Cases of fracture were not included in this study.

\section{Results}

Incidence of lameness reported in different species over the entire study period were highest in canine reported with 56\% (286 lameness cases out of total 511) followed by equine with $21 \%$ (109), cattle and caprine $7 \%$ (35), buffalo 5\% (28), feline 3\% (16), sheep $0.6 \%$ (3), monkey $0.39 \%$ (2) and swine $0.19 \%$ (1).

Canine: In dogs, the different causes for lameness were hind quarter weakness (HQW) 55\% (158/286), right hind limb lameness 15\% (42), left hind limb lameness $13 \%$ (36), left fore limb lameness 12\% (34), hip dislocation $6 \%$ (18) and hip dysplasia 4\% (12). The breeds which mostly affected by HQW were Mongrel $37 \%$ (58/158) followed by GSD 29\% (46), Spitz 20\% (32) and others $14 \%$ (22). In all the breeds except in Labrador, males were more affected than females. Mongrel reported $72 \%$ males and $28 \%$ female cases, GSD with $61 \%$ males and 39\% females and in Spitz $63 \%$ males and $37 \%$ females were affected. Most of the affected dogs were either less than one year or above 6years of age.

Forty two cases of right hind limb lameness were recorded, in which Spitz affected with 33\% (14/42) followed by GSD 14\% (6), Doberman, Mongrel, Rottweiler, Labrador 9.5\% (4), Cocker Spaniel and Neopolitan Mastiff 4.7\% (2). Except in Mongrel breed, the incidence of lameness was more in male animals. Animals below 2 years and above 8 years of age were mostly affected. A total of 36 cases of left hind limb lameness were reported, mostly in Mongrel 28\% (10/ 36). Other breeds affected were GSD 22\% (8), Labrador 17\% (6), Spitz 11\% (4), Doberman, Dalmatian and Pug $5.5 \%(2)$. Males were affected more in all breeds except GSD, Labrador and Pug. Most of animals affected were under 1 year and above 7 years of age.

Among the 34 cases of Left forelimb lameness reported, the most commonly affected breeds were GSD 47\% (16/34), Mongrel 23.5\% (8), Spitz, Labrador 
$12 \%$ (4) and Doberman 6\% (2). Males were mostly affected except in Mongrel. Most of the animals affected were above 4 years of age. Few cases of right forelimb lameness were reported in male GSD and female Spitz dogs. As much as 18 cases of hip dislocation were reported with higher incidence in GSD and Labrador 28\% (5/18) followed by, Spitz 22\% (4), boxer 11\% (2) and others $11 \%$ (2). Males were mostly affected. Bilateral cases were reported more in Spitz.. Twelve cases of Hip Dysplasia were reported among GSD, Great Dane, Pug, Labrador, Spitz and Mongrel. Right sided cases were reported more in Labrador and Spitz whereas left sided cases were more in GSD and Mongrel.

Stiffness of both the forelimbs were found in Mongrel and Spitz dogs. A case of right shoulder dislocation was reported in a female Spitz. Bilateral capped elbow was recorded in male Labrador and female Spitz. A case of radial nerve paralysis was reported in female Spitz. Few cases of lumbar spondylosis in Spitz and patellar displacement were found to be more in Spitz and Labrador. Lameness due to wound and cellulitis were also reported in different breeds. About 54 cases of rickets were recorded and more in Spitz 44\% (24), Mongrel 39\% (21) and GSD $16 \%$ (9). Most of the animals affected were below 5 months of age.

Equine: Next to canines, equines were found to have more incidence of lameness. A total of 109 cases of lameness were reported in which $17 \%$ with right forelimb affection, $13 \%$ of cases were due to right hind limb affection, $11 \%$ due to left forelimb affection, $4 \%$ due to disease of right shoulder, HQW and bilateral forelimb affection each, $3 \%$ due to lateral dislocation of patella, $9 \%$ due to affection of both hind limbs, 5.5\% due to tenosynovitis, arthritis and bone spavin, $7 \%$ due to laminitis of right hind and forelimbs and $4.5 \%$ due to quittor.

Caprine: In caprines, a total of 35 lameness cases were reported with right forelimb lameness (23\%), shoulder dislocation (20\%), right hind limb lameness (12\%), left forelimb lameness $(12 \%)$, posterior paresis $(9 \%)$ and others $(24 \%)$.

Cattle: In cattle, 35 lameness cases were recorded, of which $28.5 \%$ of cases were due to HQW. Most of the affected cows were around 2 or 5 years of age. About $34.28 \%$ of cases were due to right hind limb lameness, $8.57 \%$ with left hind limb lameness, $14.28 \%$ with hip dysplasia, 6\% with obturator nerve paralysis and $8.57 \%$ cases were due to contracted tendon in calves. Among the 28 buffalo lameness cases reported, 14.28 $\%$ of cases were due to right carpal arthritis, foot rot and left hind limb lameness, followed by $18 \%$ due to bilateral upward luxation of patella and bilateral purulent wound in stifle joint, and $21.4 \%$ due to hip dislocation.

Feline: In feline, about 16 cases of lameness were recorded, out of which $68 \%$ (11) of cases were due to HQW and rest due to dislocation of thoracic or lumbar vertebrae.

Others: Lameness due to osteoarthritis was reported in 3 sheep. Posterior paralysis due to fight among the animals were encountered in 2 male monkeys. In porcine a case of left hindlimb lameness due to wound was recorded in a one month old Large White Yorkshire male.

\section{Discussion}

The cause of lameness varies widely and is significantly influenced by the species, breed, age, sex and the nutritional status of the animal. Most important causes of lameness are classified as degenerative, developmental, metabolic, mechanical, infectious, inflammatory and traumatic. Developmental and metabolic causes of lameness are found to be more in young growing animals and degenerative causes in aged ones. Lameness is an important clinical condition in dogs especially in active energetic animals. Apart from fractures, the other important causes of lameness includes hip dysplasia, hip dislocation, nerve paralysis, vitamin and mineral deficiencies etc. Heavy and light breeds are affected by different conditions. Skeletal disorders are found to be more in large breeds of dogs that occur during the period of rapid growth up to two years of age. Lameness in hindlimb is commonly associated with the stifle (cranial cruciate ligament disease) and the hip (hip dysplasia) [8]. Diagnosis of the cause of lameness is imperative and the most critical part in treatment of lameness is management of pain and inflammation [9].Conditions like HQW are found to be more in males than in female animals and the causes are degenerative myelopathy, injury to the spinal cord, Cushing's disease, diabetes and arthritis. Hip dysplasia was found mostly in GSD breeds. Spitz breeds due to its increased activity are found to be very prone to all conditions of lameness like nerve injuries, dislocations, and wounds.

In sheep, incidence of lameness is found to be higher during wet months. Overgrown hooves and traumatic injuries are found to be the important predisposing factors [10]. In horses, incidence of lameness is found to be slightly more in forelimb than in hindlimb [11]. Common causes of lameness in horses are degenerative conditions like degenerative joint disease (DJD, or osteoarthritis), developmental abnormalities like osteochondrosis (OCD), physitis (epiphysitis), metabolic diseases like laminitis (founder), exertional rhabdomyolysis (tying up), mechanical conditions (overload), infectious causes like foot abscess, infected wound, cellulitis [12], joint infection, inflammatory and traumatic causes. Age and duration of service is not found to be significantly different between lame and non-lame population of horses [12]. In goat and cattle, systemic diseases, musculoskeletal disorders and nervine problems are found to be the main causes of lameness [13]. In cattle, 
the most important causes of lameness are laminitis, claw disease, digital dermatitis, and foot rot.

The cause of lameness should be diagnosed using X-rays, blood parameters etc to distinguish skeletal, muscular, nerve problems. The conditions like Cushing's disease, Hyper and hypothyroidism, diabetes should be ruled out and treatment should be decided accordingly. Exercise has been recommended to maintain the dog's ability to walk. Physiotherapy may prolong the length of time that the dog remains mobile and increase survival time $[15,16]$. Canine hydrotherapy (swimming) may be more useful than walking [16]. Animals were given different treatment methods like, nonsteroidal anti-inflammatory drugs, physical therapy, vitamins or minerals, steroids, acupuncture and confinement depending on the condition.

This pilot study has several limitations and is retrospective, a survey that was limited to those animals referred to Division of Veterinary Surgery, IVRI. The influence of diet and feeding status could not be assessed as this was often missing from the medical records.

\section{Conclusion}

Our study has shown that incidence of lameness in animals is significantly affected by species, breed, age and sex of the animal. Different species are susceptible to different conditions. In dogs and cats, HQW is the most common cause of lameness and in dogs breeds mostly affected were Mongrel and GSD. Male animals of less than 1year or above 6 years are affected more. Hip dislocation is found to be more in male GSD and Labrador dogs and bilateral hip dislocation in spitz breeds. Hip dysplasia is also found to be more in GSD breed. Incidence of rickets is more in Spitz dogs below 5 months of age. In equines and caprines, right forelimb lameness, and in cattle right hindlimb lameness is more. Hip dislocation, upward luxation of patella and wounds are the major lameness causes in buffaloes.

\section{Authors' contributions}

AM and MG collected the data, PT and KK prepared the manuscript. MBG and MMSZ contributed in analyzing data. KS and AG corrected the manuscript. All authors read and approved the final manuscript.

\section{Acknowledgements}

The authors are highly thankful to the Director of the Institute for providing funds and facilities to carry out this study.

\section{Competing interests}

The authors declare that they have no competing interests.

\section{References}

1. Chris Whitton (2010) Lameness in Horses: Development of orthopedic disease. The Merck Veterinary Manual (10 $10^{\text {th }}$.). Merck \& Co., Inc. and Merial Limited. p917.

2. Harry Scott and Philip Witte (2011) Investigation of lameness in dogs.Forelimb lameness. In practice 33: 20-27.

3. Ian Wright (2013) Case study: Canine Lyme disease presenting as a forelimb lameness. Companion Anim Pract 18(8):372-373

4. Roger Blowey (2005) Factors associated with lameness in dairy cattle. In practice 27:154-162.

5. Mate Zoric.,Ebba Nilsson., Nils Lundham., Per Wallgren. (2009) Incidence of lameness and abrasions in piglets in identical farrowing pens with four different types of floor. Acta Vet Scand.51:23.

6. Michael A. Hill (2010) Lameness in Pigs. Overview of lameness in pigs. The Merck Veterinary Manual $\left(10^{\text {th }} \mathrm{ed}\right)$. Merck \& Co., Inc. and Merial Limited. p1047.

7. David Weaver, Guy St. Jean, Adrain Steiner. (2005) Bovine Surgery and Lameness. Blackwell publishing $\left(2^{\text {nd }} e d\right)$ p199200.

8. Levine, J.M., Hillmann, R.B., Erb, H.N., de Lahunta, A. (2002) The influence of age on the patellar reflex response in the dog. J Vet Intern Med.16:244-246.

9. Harry Scott and Philip Witte (2011) Investigation of lameness in dogs. Hindlimb lameness. In practice. 33: 5866.

10. Kim Henneman (2013) Winter Lameness. IVC Journal.3:1.

11. Bokko, B. P., Adamu, S. S., Mohammed, A. (2003) Limb condition that predispose sheep to lameness in the arid zone of Nigeria. Small Ruminant Res 47(2): 165-167.

12. Keegan,K. G., Dent, K. V., Wilson, D. A., Janicek, J., Kramer, J., Lacarrubba, A., Walsh, D. M., Cassells, M. V., Esther, T. M., Schiltz, P., Frees, K. E., Wilhite, C. L., Clark, J. M., Pollitt, C. C., Shaw, R., Norris, T. (2010) Repeatability of subjective evaluation of lameness in horses. Equine Vet $J$. 42(2):92-97.

13. Putnam, J. R. C., Holmes, L. M., Green, M. J., Freeman, S. L. (2014) Incidence, causes and outcomes of lameness cases in a working military horse population: A field study. Equine Vet J. 46(2):194-197.

14. Michael A. Hill (2010) Lameness in Pigs. Overview of lameness in pigs. The Merck Veterinary Manual $\left(10^{\text {th }}\right.$ ed.) . Merck \& Co., Inc. and Merial Limited. p1047.

15. Kahn, Cynthia M., Line, Scott, eds. (2005) Degenerative Diseases. The Merck Veterinary Manual (9th Edn.). Merck. ISBN 0-911910-50-6.

16. Kathmann, I. I.,Cizinauskas, S., Doherr, M.G., Steffen, F., Jaggy, A. (2006) Daily controlled physiotherapy increases survival time in dogs with suspected degenerative myelopathy. JVet Intern Med. 20 (4): 927-932. 